\title{
¿Es viable implementar la renta básica universal en Colombia?
}

\author{
AngÉlica Olave Gutiérrez ${ }^{*}$
}

\section{RESUMEN}

Este artículo tiene como objetivo analizar el debate presentado en Colombia sobre la posible implementación de la renta básica universal, entendida esta como una medida que busca garantizar un ingreso mínimo para las personas que no pueden acceder al mercado laboral. Así las cosas, en primera medida se explicará el contexto en el que surgió la propuesta en Colombia (la pandemia de la covid-19), luego estudiaremos la experiencia de otros países y, por último, analizaremos si en el contexto económico, político y social actual del país hay cabida para la aplicación de esta medida.

Palabras clave: renta básica universal, covid-19, subsistencia mínima, renta básica garantizada.

\section{¿IS IT FEASIBLE TO IMPLEMENT UNIVERSAL BASIC INCOME IN COLOMBIA?}

\section{ABSTRACT}

This paper aims to analyze the debate presented in Colombia on the possible implementation of the universal basic income. It is understood as a measure that seeks to guarantee a minimum income for people who cannot access to the labor market. Thus, first we will explain the context in which the proposal arose in Colombia (The covid-19 pandemic), then we will study the experience of other countries and finally, we will analyze if in the current economic, political and social context of the country, it is possible to apply this policy.

Keywords: Universal basic income, covid-19, minimum subsistence, minimum guaranteed income.

1 Abogada de la Universidad Libre de Colombia (2017). Especialista en Responsabilidad Civil y Daño Resarcible de la Universidad Externado de Colombia (2019). Candidata a magíster en Derecho Económico de la Universidad Externado de Colombia.

* DOI: https://doi.org/10.18601/01236458.n55.04 


\section{INTRODUCCIÓN: CONTEXTO ECONÓMICO SOBRE LA IMPLEMENTACIÓN DE LA RENTA UNIVERSAL BÁSICA EN LOS TIEMPOS DE COVID-19}

Actualmente, el mundo entero enfrenta uno de los retos más importantes de los tiempos modernos, la llegada del nuevo coronavirus covid-19 ha puesto a prueba la adaptabilidad de los ordenamientos jurídicos frente a las situaciones de crisis. El brote masivo de este virus logró conquistar la esfera internacional, esto de tal manera que la Organización Mundial de Salud (OMS) determinó que el contagio producido por la covid-19 hacia caso a una pandemia, esto es, una afectación masiva de personas por la propagación de un virus en un área geográficamente extensa.

Desde la perspectiva internacional, la gran mayoría de países han tenido que aplicar medidas preventivas, esto con el fin de aminorar las cargas de los sistemas de salud y proteger la vida de las personas. A la fecha de este artículo (octubre de 2020), a nivel mundial, cerca de cuarenta millones de personas ya se habían contagiado de la covid-19 y a causa de la misma enfermedad murieron más de 1,1 millones personas en todo el mundo (Universidad Johns Hopkins, 2020).

En este contexto, una de las medidas de mayor implementación a nivel mundial para la prevención del contagio ha sido el confinamiento o aislamiento, entendido este como el distanciamiento social de las personas en sus hogares. Esta ha sido la principal herramienta de la administración pública frente al problema que genera la covid-19 como un virus de contagio masivo.

En el caso de Colombia, el presidente de la República decretó el Estado de Emergencia Económica, Social y Ambiental por medio del Decreto Legislativo 417 del 2020, en esta disposición se enervaron las facultades que le otorgan los artículos 212 y 213 de la Constitución Política, lo que permite la existencia de un estado de excepción que faculta al presidente, como máxima autoridad administrativa, a ejercer potestades especiales para emitir decretos con fuerza de ley (Presidencia de la Repúbica de Colombia, 2020).

Con base en lo anterior, el Ministerio de Interior emitió el Decreto Legislativo 457 de 2020 (Presidencia de la República, 2020), el cual en su artículo 1 decretó el aislamiento preventivo obligatorio, esto con el fin de salvaguardar la vida e integridad de las personas que puedan ser infectadas por el nuevo coronavirus covid-19.

Ahora, muchas cosas se han dicho respecto de la idoneidad y proporcionalidad que tiene el aislamiento para enfrentar los altos niveles de contagio que puede ocasionar la covid-19, pero lo cierto es que dicha medida ha sido una herramienta útil para crear un umbral de acción en favor de la administración pública, esto en lo que tiene ver con medidas adicionales como el testeo masivo de personas y el reforzamiento o acondicionamiento del sistema de salud.

No obstante, el aislamiento preventivo obligatorio tiene varios resultados indeseados que al día de hoy se han ido descubriendo y que, para el caso de Colombia, se han ido tratando con los decretos legislativos expedidos por la Presidencia de la República. Así, una de las áreas del país más afectada por el confinamiento es la economía, ya que 
ello implica una desactivación de todos los procesos productivos que requieren de presencialidad y altos niveles de trabajo humano para continuar operando. De hecho, a pesar del abrumador crecimiento de la tecnología, actualmente la producción de varios bienes y servicios no puede ser desarrollada a través de la virtualidad, sino que resulta indispensable la presencia de las personas en sus lugares de trabajo, esto es, claramente, un riesgo en épocas de pandemia.

Una de las formas de evidenciar el efecto negativo del confinamiento y, por ende, la afectación de la producción nacional se encuentra en los índices de desempleo y crecimiento. En efecto, a la fecha de este artículo, y según el último informe del Departamento Administrativo Nacional de Estadística (DANE), para el mes de agosto de 2020, la tasa de desempleo asciende al 16,8 \%, lo cual significó un aumento de seis puntos respecto del mismo mes del 2019. Adicionalmente, teniendo en cuenta el mismo informe, solo el 49,3 \% de la población se encuentra ocupada en una actividad laboral formal o informal (Departamento Administrativo Nacional de Estadística [DANE], 2020).

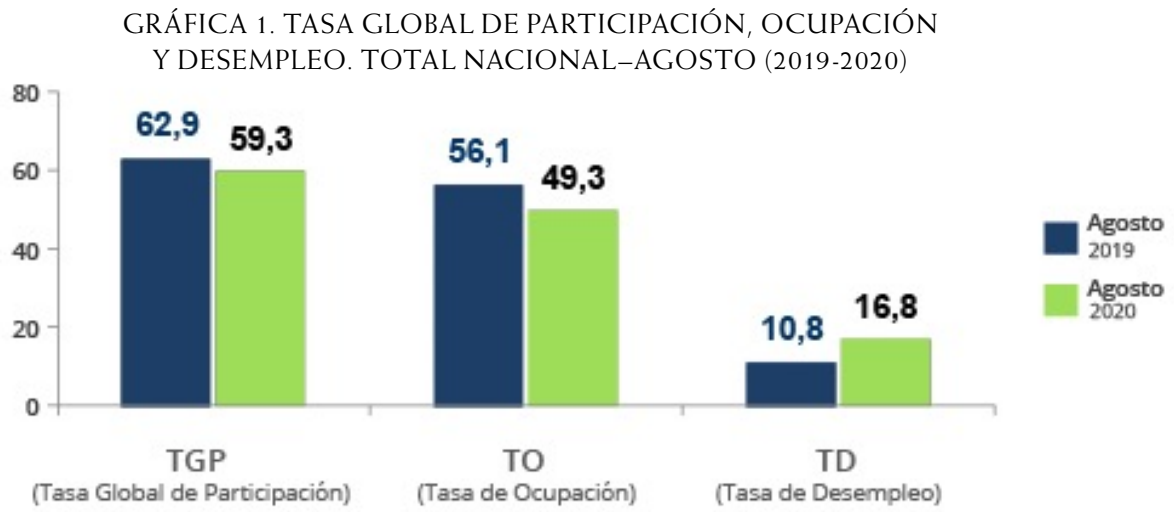

Fuente: Departamento Administrativo Nacional de Estadística-DANE (2020).

Un informe de la Universidad de los Andes de octubre de 2020 muestra un diagnóstico desalentador. Entre otros, se menciona que entre el segundo trimestre de 2019 y 2020 se perdieron cerca de 4,9 millones de empleos en el país; en el mismo periodo, la proporción de hogares cuyos ingresos totales son menores a un salario mínimo pasó de $34 \%$ a $48 \%$ y, por primera vez, la proporción de hogares que reportaron cero ingresos ascendió a 10 \% (Universidad de los Andes, 2020a).

Por otro lado, en lo que tiene que ver con el crecimiento de la economía, el Fondo Monetario Internacional redujo la proyección del crecimiento colombiano de 3,6\% a 3,5\% para la presente anualidad (Ramírez Bonilla, 2020). Sin embargo, para el primer trimestre de 2020, el producto interno bruto (PIB) colombiano tenía una variación positiva de 3,4\% respecto del periodo inmediatamente anterior. Pero en el último informe del Banco de República, este mismo índice solo tiene una variación de 0,4\%, cifra que 
jamás había estado por debajo de uno, esto teniendo en cuenta los datos recopilados desde el primer trimestre de 2005 (Banco de la República de Colombia, 2020).

Dicho lo anterior, la disminución del empleo y el crecimiento ha puesto en evidencia una realidad social, esto en lo que tiene que ver con la subsistencia mínima de muchos hogares colombianos. Recordemos que para mediados de 2020, en Colombia la tasa de empleo informal era del 46,4\% (Departamento Administrativo Nacional de Estadística [DANE], 2020) y este tipo de empleo se constituye de ocupaciones que, según la misma metodología del DANE, están relacionadas con trabajos de jornaleros y peones, así como trabajadores por cuenta propia, incluyendo aquellos que se dedican al mercado callejero, empleados domésticos y el comercio básico, por tanto, se trata de trabajadores que en su mayoría no pueden compatibilizar su trabajo con las medidas de aislamiento preventivo (p. ej., teletrabajo). Sobre este punto, según una estimación de economistas del Departamento Nacional de Planeación, de los dieciséis millones de trabajadores habilitados en Colombia, alrededor de nueve millones (56,4\% del total) no podrían trabajar desde sus casas, se trata de trabajadores que necesariamente deben movilizarse a sus lugares físicos de trabajo (Lamprea, Ospina, Hernández y Rivera, 2020).

Respecto de la estimación del impacto sobre los trabajadores, con una caída del $-16,8 \%$ al $-22,9 \%$ del empleo informal entre el segundo trimestre de 2019 y de 2020, este empleo fue de los más golpeados junto con empleos en sectores no esenciales $(-25,7 \%)$, asalariados $(-23,1 \%)$ y empleos en empresas de menos de 10 trabajadores (-24,3\%) (Universidad de los Andes, 2020b). Esto en definitiva denota que los empleos en el sector informal y en pequeñas empresas son altamente vulnerables por efecto de la pandemia y de las mismas medidas gubernamentales (Eslava y Isaacs, 2020).

De esta manera, nótese que se presenta entonces una suerte de paradoja social expresable en que por efecto del aislamiento preventivo ordenado por el Gobierno nacional durante la pandemia, aquellas personas que se encontraban dedicadas a dichas actividades informales y en pequeñas empresas se les exige que cumplan con una suerte de "compromiso social" de mantenerse aisladas, para de esta manera reducir las tasas de contagio y así alivianar la carga del sistema salud, pero, al mismo tiempo, aquellas son las personas que ven drásticamente reducidos sus ingresos diarios, los cuales nunca, menos aún durante la pandemia, han sido suficientes para la constitución de ahorros, variable fundamental a la hora de enfrentar la medida de aislamiento.

En este orden de ideas, era previsible que el debate político nacional se hubiese concentrado en el mayor impacto que el aislamiento produce sobre los sectores laborales informales, por lo mismo, desde el inicio de la pandemia, se dio un consenso de concentrar las ayudas económicas en este tipo de trabajadores y sus familias, que en conjunto representan unos 23 millones de personas (Universidad de los Andes, 2020b).

En este contexto surgió nuevamente el debate en torno a la posibilidad de aprobar, por vía legislativa, una renta básica universal, lo que significa garantizar un ingreso mínimo para las personas en situación de pobreza que acrediten la imposibilidad de devengar un ingreso por sí mismos. Por supuesto, la implementación de esta política 
pública no es asunto fácil, pues posee un sinnúmero de aristas, así como detractores y defensores que no solo discuten por el presupuesto público que requiere este tipo de medidas, sino también por la adecuada focalización de los destinatarios de este programa, donde, entre otras, es necesario prevenir cualquier incentivo a no conseguir empleo, así como evitar efectos inflacionarios.

Así las cosas, luego de explicar un breve contexto macroeconómico en que se desenvuelve el debate de la renta básica universal, a continuación, mencionaremos la experiencia internacional con la implementación de esta medida, esto para después analizar su posible aplicación en Colombia y, por último, llegar a unas conclusiones muy preliminares a manera de ensayo.

\section{RENTA UNIVERSAL BÁSICA EN LA EXPERIENCIA INTERNACIONAL}

En general, se puede decir que la denominada "renta básica universal" o universal basic income (también llamado "ingreso básico garantizado" [IBG] o basic income guarantee [BIG]) responde a situaciones relativamente similares, con todo y que la experiencia en diferentes latitudes se ha dado con presupuestos y resultados diferentes. No obstante, el principal problema, el foco de la discusión, que trae consigo esta figura, se relaciona con las dificultades fiscales que supone su implementación para cualquier país, lo que es un aspecto especialmente crítico en aquellos países menos desarrollados en comparación con aquellos más desarrollados (Hoynes y Rothstein, 2019).

Ahora, a nivel internacional y según algunos autores, la renta básica universal es una figura que surge en el marco del Estado del bienestar (Van Parijs, 1992), lo cual supone que es entonces parte de la faceta prestacional de una nación y a través de esta prestación se señala que es posible mejorar las condiciones de vida de las personas y satisfacer sus necesidades básicas. De esta manera, esta es una idea que suele compatibilizarse con la implementación del Estado social de derecho y los derechos sociales subjetivos que se derivan de él (De Wispelaere y Morales, 2016a). Al respecto, si bien desde una perspectiva constitucional tales derechos sociales no son absolutos (y la renta básica universal no puede ser entonces la excepción), lo cierto es que con el mismo tipo de argumento "social" se han fundamentado otras políticas como aquellas que tienen por objeto garantizar la prestación y continuidad de los servicios públicos o aquellas otras relacionadas con los "mínimos vitales" que como tal suelen concentrarse y dirigirse a los sectores sociales económicos más vulnerables (De Wispelaere y Morales, 2016b, García, 2005).

A pesar de lo anterior, es importante tener en cuenta que esta faceta prestacional que caracteriza al Estado de bienestar o al Estado social de derecho no tiene por qué suponer una cobertura o efectividad total de la prestación a cargo del mismo Estado, por ejemplo, en lo que se refiere a servicios públicos domiciliarios, la regulación en algunos países ha optado por implementar modelos de mercado en los que los prestadores cobran tarifas a los usuarios a cambio del servicio y, en algunos otros casos, para completar la cobertura del servicio respecto de toda la población operan subsidios 
obligatorios a favor de los mismos usuarios. Similar discusión respecto de la faceta prestacional del Estado social, se da en relación con la provisión de prestaciones sociales que son cubiertas parcialmente a través de mecanismos de mercado, mientras que otros segmentos de la población reciben subsidios, tal como ocurre con la prestación del servicio de salud, el transporte público, la educación, o incluso a veces subsidios al empleo y a las pensiones (Phelps, 1994).

El asunto de la renta básica universal se enfrenta a una discusión bastante similar al de cualquier otra faceta prestacional del Estado de bienestar o del Estado social de derecho, en el que el asunto del presupuesto público desempeña un papel indispensable ${ }^{2}$, máxime cuando la mayoría de estos servicios "sociales" no pueden dejarse totalmente en manos de particulares para que estos garanticen su prestación, ya que esta prestación es imposible garantizarla a través de una situación de libre mercado.

Ahora, aunque existen algunas primeras experiencias en el siglo XVI, el debate contemporáneo sobre la implementación de programas sociales de renta básica universal inicia con la experiencia de algunos países europeos entre finales de los años de 1970 y comienzos de 1980, ello con la particularidad de que en la mayoría de los casos no se dio por medio de una política nacional, sino que fueron acciones administrativas de gobiernos locales o provinciales, quizá con el fin de lograr una mejor focalización y control de la medida. A manera de ejemplo, desde inicios de los años de 1980 se creó el Alaska Permanent Fund, un fondo de inversión financiado por recursos de la renta petrolera y mediante el cual el Gobierno de Alaska provee un dividendo de USD 6600 al año a cada persona que lleve viviendo más de seis meses en ese Estado (Berman, 2018, Van Parijs y Vanderborght, 2015, pp. 46 y ss.).

Posteriormente se han dado otras experiencias de renta básica universal en las provincias canadienses de Ontario y Manitoba, así como también en otros estados de Estados Unidos como California, Indiana, Carolina del Norte, regiones cheroquis; la región de Macau en China, las regiones de Odisha y Nueva Delhi en India, así como en Barcelona en España. A nivel de países, el primero de ellos donde se iniciaron las primeras experiencias de renta universal fue Países Bajos, y hoy día existen programas similares en países bastante disimiles como Italia, Irán, Mongolia, Kenia, entre otros (Gentilini, Grosh, Rigolini y Yemtsov, 2020, pp. 22 y ss.).

Sin perjuicio de estas experiencias, es importante mencionar que la figura objeto de estudio está lejos de ser una política generalizada y afianzada incluso en los Estados sociales de derecho y en su lugar lo que existen son múltiples experimentos que permiten evidenciar tanto los resultados positivos como negativos. En el caso de Holanda, por ejemplo, el plan piloto se realizó en la ciudad de Utrecht, donde los subsidios se otorgaron a una población que ya había recibido algún tipo de ayuda estatal, lo que pone en duda la efectividad del experimento, ya que no se hizo un estudio independiente para su implementación. En Canadá, en la ciudad de Ontario, uno de los objetivos

2 Véase Corte Constitucional, Sentencia de Tutela 406, de 5 de junio de 1992, M. P. Ciro Angarita Barón. 
del plan era determinar cuántas personas abandonan la búsqueda de un empleo. En lo que tiene que ver con la ciudad de Barcelona, el proyecto implementado se llamaba B-Mincome y, al igual que Utrecht, las ayudas se distribuyeron a las personas que ya habían sido usuarias de servicios sociales (Tena Camporesi, 2018).

En cada uno de los experimentos mencionados, ya sea de manera principal o secundaria, el objetivo era diagnosticar qué sucedería con el mercado laboral, por ejemplo, si las personas dejaban de trabajar o cambiaban de trabajo. Entonces, al fin y al cabo, la renta básica universal tiene una incidencia en el ingreso de las personas, de manera que es bastante probable que algunos beneficiarios de un salario mínimo prefieran continuar recibiendo dicho ingreso por parte de un subsidio y dejar de trabajar. Pero no se puede dejar a un lado que la demanda de trabajo no se iguala a la oferta, de modo que el mercado dejará a muchas personas por fuera sin la posibilidad de obtener un ingreso que les permita la subsistencia.

Es así como podemos evidenciar que algunos de los principales problemas de la renta universal radican en tres puntos: (i) su presupuesto y sostenibilidad fiscal, (ii) los desincentivos que genera para la búsqueda de empleo y (iii) la subsistencia mínima de las personas que no pueden acceder al mercado laboral.

Dicho esto, y en el contexto creado por la pandemia de la covid-19, a continuación, pasaremos a analizar el debate de la posible implementación de la renta básica universal para el caso de Colombia, donde la problemática mencionada puede darse con base en los presupuestos estudiados.

\section{RENTA UNIVERSAL BÁSICA EN COLOMBIA}

El contexto actual de la discusión sobre la renta básica universal en Colombia se da a propósito de las medidas tomadas por el Gobierno nacional en el marco del estado de emergencia económica, social y ambiental (Decreto Legislativo 417 del 2020 y posteriores que lo prorrogaron). Dentro estas medidas, el mismo gobierno, a través del Decreto 518 de 4 de abril de 2020, creó el programa de Ingreso Solidario, con el fin de "[... atender las necesidades de los hogares en situación de pobreza y vulnerabilidad en todo el territorio nacional, en el marco del Estado de Emergencia Económica, Social y Ecológica". Este programa pretende brindar cobertura a aquellos ciudadanos que no se encontraban cobijados por otros programas similares y solo por el tiempo que perduren las causas que motivaron la declaratoria del mencionado estado de emergencia económica, social y ecológica.

Como se evidencia en el mismo Decreto 518 de 2020, en efecto, son varios los programas sociales y las estrategias de transferencias monetarias que se implementaron o que se reforzaron por el Gobierno nacional, al menos durante la primera fase de la pandemia de la covid-19. Entre estas estrategias se resaltan especialmente: (i) una transferencia extraordinaria de \$ 145000 para cerca de 2,6 millones de hogares beneficiarios del programa Familias en Acción, (ii) una transferencia extraordinaria de cerca de \$ 356000 para las 206 mil personas del programa Jóvenes en Acción; (iii) 
un adelanto de las transferencias a 1,7 millones de inscritos en el programa Colombia

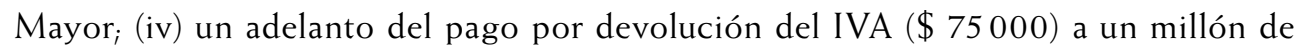
beneficiarios de menores ingresos (conforme el Plan Nacional de Desarrollo 2018 2020); (v) la implementación del programa Ingreso Solidario (Decreto legislativo 518 del 4 de abril de 2020) que supone una transferencia de $\$ 160$ mil pesos para más de tres millones de familias no cubiertas por otros programas, y que en el segundo mes de confinamiento se planteó de ochenta mil pesos (Sánchez y Chaparro, 2020, pp. 12-13).

Por otro lado, vale mencionar que un grupo de 54 senadores de la República radicó el proyecto de Ley 310 de 2020/Senado ${ }^{3}$ con el cual se pretendía modificar el decreto legislativo en mención, ampliando su cobertura y aumentando el monto de las transferencias hasta alcanzar un salario mínimo legal mensual vigente. Aunque dicho proyecto no es el único que pretende regular la materia y además fue archivado, este sí animó aún más la discusión sobre la conveniencia de la implementación de un programa de transferencias monetarias que encaje dentro de la idea de renta básica universal en el país. Vale decir, además del Gobierno nacional y el Congreso de la República, de esta discusión hicieron parte economistas, centros de pensamiento y algunas universidades como la Universidad de los Andes, la Universidad Nacional de Colombia y la Pontificia Universidad Javeriana.

Así, por ejemplo, el mencionado estudio de Sánchez y Chaparro (2020) indica que aun con las citadas transferencias monetarias y las medidas tomadas por el Gobierno nacional colombiano durante la pandemia, se estimaba que para mediados de 2020, la pobreza en el país se habría incrementado en un 78 \% y la pobreza extrema se habría multiplicado por 4, lo cual supone que una sexta parte de la población colombiana pasaría a tener un ingreso tan bajo que ni siquiera sería suficiente para el consumo de los alimentos básicos. El mismo estudio indica que las transferencias efectuadas por el gobierno tienen tan solo un efecto marginal en la reducción de la pobreza, en concreto se indica que, después de un mes de confinamiento, dichas medidas reducen únicamente dos puntos porcentuales la pobreza (34\% a $32 \%)$ y 2,3 puntos la pobreza extrema (19\% a $16,7 \%)$.

Por supuesto, incrementar el monto de las transferencias monetarias tiene un impacto en el presupuesto público, sin embargo, esta afirmación es necesario hacerla en perspectiva. Según Sánchez y Chaparro (2020), el costo actual de las transferencias del programa Ingreso Solidario (Decreto legislativo 518 de 4 de abril de 2020) equivale a tan solo el 0,16\% del presupuesto público y al 0,04\% del PIB. En contraste, en países, como Brasil, el mismo tipo de transferencias representan el 1,2 \% del PIB. Al tiempo, cabe contrastar que los recursos económicos destinados por el mismo

3 Senado de la República de Colombia. Véase en http://leyes.senado.gov.co/proyectos/index.php/ proyectos-ley/cuatrenio-2018-2022/2019-2020/article/310-por-medio-del-cual-se-modifica-el-decreto-ley-518-de-2020-por-el-cual-se-crea-el-programa-ingreso-solidario-para-atender-las-necesidadesde-los-hogares-en-situacion-de-pobreza-y-vulnerabilidad-en-todo-el-territorio-nacional-en-el-marco-del-estado-de-emergencia-economica-social-y-ecologica-y-se-crea-la-renta-basica-de-emergencia 
Gobierno colombiano para respaldar los créditos de las empresas durante la crisis representan alrededor del $7 \%$ del PIB (Garay y Espitia, 2020). Se estima que, si las transferencias monetarias a las mismas familias más pobres se incrementaran a un salario mínimo, el impacto sería de un 0,91\% del presupuesto público y 0,23\% del PIB (Sánchez y Chaparro, 2020, pp. 16-22), lo cual todavía estaría muy por debajo de lo que habrían recibido las empresas durante la misma crisis. Con todo, pareciera que, no obstante, con el alto impacto en el presupuesto público no solo es posible incrementar las transferencias monetarias a los sectores más vulnerables (conforme las diferentes líneas de pobreza), sino que además se abre la puerta a la factibilidad permanente de una "renta básica en Colombia".

Es importante advertir que la posibilidad de implementar una "renta básica universal" en Colombia no puede analizarse como una medida aislada ni como algo novedoso en el país, por un lado, porque las políticas de subsidios en áreas como los servicios públicos domiciliarios, las pensiones o el servicio de salud podrían apalancarse o fusionarse con una "renta básica universal" y, por otro, porque programas sociales que ya estaban establecidos incluso antes de la pandemia de la covid-19, como la "devolución del IVA", "Familias en Acción", "Jóvenes en Acción", "Colombia Mayor", ya funcionaban a través de transferencias monetarias a los más vulnerables, lo cual supone que estos también tienen elementos comunes, luego también podrían incluso reemplazarse y quedar subsumidos dentro una eventual programa de renta básica universal. Por tanto, los recursos dirigidos actualmente a subsidios y las transferencias monetarias de los programas vigentes mencionados no supondría un gasto adicional al que ya se está efectuando, pues se sumaría al presupuesto dirigido a una renta básica universal.

De acuerdo con lo anterior, un estudio de la Universidad de los Andes (2020c) asume que, si el mínimo vital se supone equivalente a la línea de pobreza monetaria, es decir, \$280000 mensuales por persona, la renta básica correspondería al monto que le falta a cada persona para alcanzar ese valor. Si se asume, en el peor escenario, 22,5 millones de personas inactivas o desempleadas, el costo anual de transferencias para completar la renta básica sería del 7,5\% del PIB. Dado que el costo actual de las transferencias monetarias vigentes es del orden del 0,3\% del PIB, los subsidios a los servicios públicos representan el 0,4\% del PIB y los subsidios a las pensiones suman el 2,5\% del PIB, entonces se necesitarían 4,3\% de ingresos adicionales para financiar una renta básica universal. Por tanto, dice el mismo estudio que para implementar este programa es necesario adelantar una reforma tributaria y mejorar las estrategias de recaudo de impuestos, además se recomienda acompañar la renta básica universal con una política de flexibilización del salario mínimo, pues la rigidez de este impide que se logre un mayor dinamismo económico, esto último es una condición para que en largo plazo se reduzca el gasto dirigido a financiar la renta básica universal.

Sobre el mismo punto, el Observatorio Fiscal de la Pontificia Universidad Javeriana, en su informe $n .^{\circ} 10$ (2020), analizó el costo y la factibilidad en diferentes escenarios de la implementación de la renta básica en Colombia, en el cual se dieron las siguientes conclusiones: 
(i) Según el Observatorio, y conforme la definición de Basic Earth Network, la renta básica universal se entiende como una asignación pública monetaria, incondicional a toda la población, consiste entonces en una transferencia de dinero que realizaría el gobierno a todos los ciudadanos mayores de 18 años, sin ningún tipo de condición especial, lo cual implicaría inicialmente incluir cerca de 36 millones de personas como beneficiarias de este programa, ello, de acuerdo con las cifras suministradas por el Departamento Administrativo Nacional de Estadística (DANE) ${ }^{4}$. Sin embargo, este primer escenario no sería viable, entre otros factores, por su alto costo para el Estado. En ese sentido, el informe presenta otros escenarios para establecer la viabilidad de este beneficio. Veamos:

[...] el primero implicaría una renta básica equivalente a la línea de pobreza extrema, en la que se entregarían $\$ 125.926$ mensuales; le costaría al país $\$ 54,5$ billones.

Un segundo escenario calcula la renta en la línea de pobreza ( $\$ 280.000$ mensuales) y tendría un costo de $\$ 119$ billones.

En un tercer escenario se tienen en cuenta los hogares donde padre y madre están en situación de pobreza y, por tanto, serían dos rentas en el hogar. Por dos rentas por casa, el costo para el país sería de $\$ 166,7$ millones.

Un cuarto y quinto escenario plantean una renta de medio salario mínimo y de un salario mínimo, respectivamente. En el primer caso, el costo anual sería de $\$ 212,3$ billones y para el segundo sería de $\$ 424,6$ billones.

Este último caso equivaldría al 40\% del PIB, lo que lo hace extremadamente difícil.

En este sentido, el estudio afirma que la renta básica universal de un salario mínimo para 9 millones de colombianos es inviable. (Revista Dinero, 2020)

(ii) La renta básica garantizada prevé un aumento en los impuestos a las personas que cuentan con altos ingresos para que ese dinero pueda transferirse a aquellos que se encuentran en condiciones de pobreza y pobreza extrema, alrededor de 3626815 personas, es decir un 7,2 \% de los colombianos vive en condiciones de pobreza extrema (Departamento Administrativo Nacional de Estadística [DANE], 2018), así las cosas, el estudio concluye que

4 Departamento Administrativo Nacional de Estadística (DANE), Proyecciones y retroproyecciones de población, véase https:/www.dane.gov.co/index.php/estadisticas-por-tema/demografia-y-poblacion/ proyecciones-de-poblacion 
Incluso si no tuvieran ningún ingreso adicional, transferirles a estos 3,6 millones de personas un monto mensual igual a la línea de pobreza extrema tendría un costo anual de $\$ 5,5$ billones de pesos. Si además se les pudiera transferir únicamente el faltante para superar la línea de pobreza, el costo sería menor. Es decir: una renta básica garantizada que acabara con la pobreza extrema en Colombia podría costar menos de $\$ 5,5$ billones al año, mucho menos que el costo de una renta básica universal del mismo monto. (Observatorio Fiscal de la Pontificia Universidad Javeriana, 2020)

Para tener una referencia sobre el costo estimado por el Observatorio Fiscal de la Pontificia Universidad Javeriana de una renta básica calculada sobre la línea de pobreza extrema, los $\$ 5,5$ billones al año equivalen al $10 \%$ del rubro de inversión o menos del 3 \% de los gastos de funcionamiento incluidos en el Presupuesto General de la Nación para el 2021.

Como se puede constatar, podríamos concluir que respecto al presupuesto y la sostenibilidad fiscal de la renta básica universal esta sería económicamente viable, sin embargo, resulta necesario resaltar que los recursos para sostener el beneficio de la renta básica garantizada son los impuestos que son recaudados y canalizados por medio del sistema tributario que, a su vez, tiene alrededor de 250 regímenes tributarios. En otras palabras, estos regímenes representan exenciones y beneficios para los contribuyentes, lo que se traduce en menos recaudo. Pero, además, de acuerdo con el análisis realizado por la Organización para la Cooperación y el Desarrollo Económico (OCDE) respecto a la recaudación tributaria sobre el Producto Interno Bruto (PIB) que en promedio en la región fue de 22,8 \%, Colombia es uno de los países de la región que tiene menor recaudo en relación con su PIB con un 19,4\%, tal y como se muestra en la gráfica 2, lo cual deja en evidencia que implementar un sistema de renta básica garantizada sería muy ambicioso y poco sostenible de acuerdo con los ingresos tributarios actuales del país. Como sugiere el estudio de la Universidad de los Andes (2020c), la implementación de un programa de renta básica universal no se puede lograr sin a su vez incluir una reforma tributaria.

Ahora, otro de los problemas prácticos de la aplicación de una renta básica universal tiene que ver con su focalización, lo cual tiene que ver con que es necesario que el Estado conozca los datos exactos de los ingresos de todos los habitantes del país, lo cual, pese a los avances que se han dado (p. ej., fase 4 del Sisbén), hoy no es posible, ello teniendo en cuenta que — como se dijo- más o menos la mitad de la población cuenta con un trabajo informal, luego el conocimiento sobre su real situación socioeconómica es bastante opaca. Esta asimetría de información sería entonces un obstáculo adicional para hacer los cálculos exactos de quienes serían los beneficiarios de una renta básica universa y del valor que debería transferirse a cada uno. 
GRÁFICA 2. RELACIÓN DE INGRESOS TRIBUTARIOS Y EL PIB (INGRESOS TRIBUTARIOS TOTALES COMO \% DEL PIB) EN LOS PAÍSES ALC, 2017

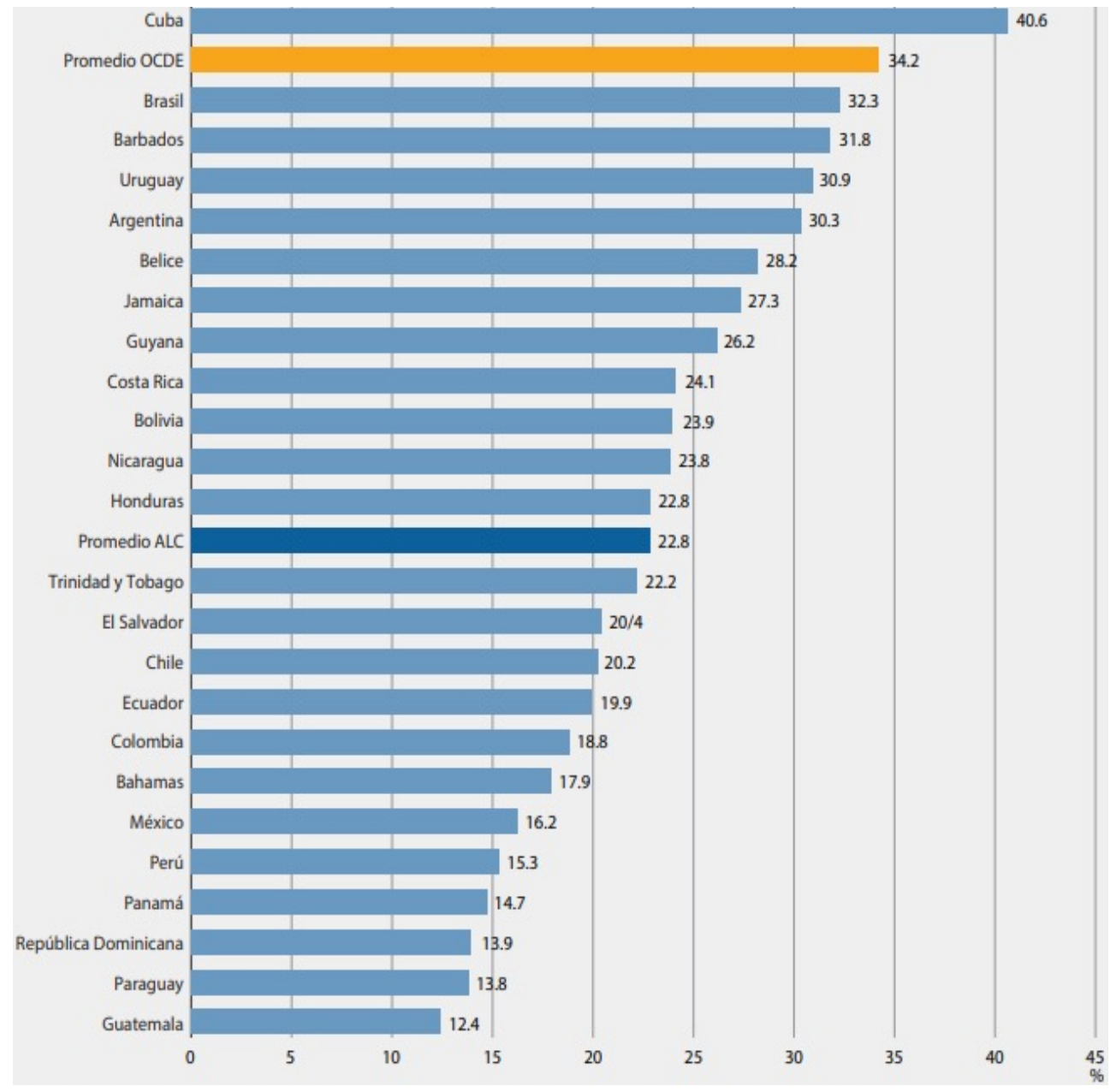

Fuente: Organización para la Cooperación y el Desarrollo Económico (2019).

Estos problemas de focalización tradicionalmente han sido solucionados con el sistema de puntaje del Sisbén y la medición de los niveles de pobreza monetaria y desigualdad realizada por el DANE, pero como ha sido ampliamente demostrado, en especial, en el marco de esta pandemia y de esta crisis económica naciente, estos datos, en muchos casos, no son fiables y llevan a que en varios casos se entreguen ayudas y beneficios a personas que no los necesitan, por tanto, se generarían incentivos para el ocultamiento de información por parte de los beneficiarios de la renta básica, así como desincentivos para la búsqueda de un mejor empleo. 


\section{CONCLUSIONES}

La primera conclusión que salta a la vista es que sí es viable para el Estado colombiano implementar y garantizar la renta básica universal, sin embargo, su implementación no puede contemplarse como un algo aislado de los demás programas de subsidios y programas sociales existente. Por otro lado, también es importante recalcar que la sostenibilidad en el tiempo de un programa de esta naturaleza se ve limitada por la capacidad de recaudo de impuestos del Estado que, como se demostró someramente, hoy tiene diversas dificultades por el sistema tributario que actualmente tiene el país, ya que, en términos económicos, el recaudo es erosionado, por lo cual con el sistema tributario actual, fiscal y presupuestalmente no sería viable.

En el mismo sentido, también se señaló que no existe información suficiente y completa para establecer quiénes son las personas que cumplen con la condición de estar en el umbral de pobreza o pobreza extrema, lo cual es de vital importancia para establecer cuál es el valor que necesita cada persona para llegar a la renta básica deseada, aun así, este problema de focalización ha sido resuelto a través del sistema de puntaje del Sisbén que, sin embargo, mantiene diversas deficiencias (que también se han ido resolviendo), por lo cual la asimetría de información tendría algunos efectos no deseados como desincentivar la búsqueda de empleo.

Sin embargo, teniendo en cuenta que para el mes de agosto de 2020 la tasa de desempleo ascendía al 16,8 \% (Departamento Administrativo Nacional de Estadística [DANE], 2020) y continúa en aumento, resulta evidente que la demanda de fuerza de trabajo continúa a la baja, de modo que el mercado laboral (formal e informal) está dejando a muchas personas por fuera, sin la posibilidad de obtener un ingreso mínimo o básico, por lo que el Gobierno deberá examinar si para contrarrestar este efecto es necesario diseñar una política pública que permita el acceso a una renta básica garantizada a la población más vulnerable, de la mano, por ejemplo, con choques tributarios, de crédito, de gasto público y programas de empleo que ayuden a disminuir los efectos no deseados de este beneficio, pero que permitan cubrir las necesidades mínimas de los colombianos.

\section{REFERENCIAS}

Banco de la República de Colombia. (2020). Boletín de indicadores económicos. https://www. banrep.gov.co/sites/default/files/paginas/bie.pdf

BERMAN, M. (2018). Resource rents, universal basic income, and poverty among Alaska's Indigenous peoples. World Development, 106, 161-172.

De Wispelaere, J. y MORAlES, L. (2016a). Is there (or should there be) a right to basic income? Philosopby \& Social Criticism, 42(9), 920-936.

De Wispelaere, J. y Morales, L. (2016b). The stability of basic income: A constitutional solution? Journal of Public Policy, 36(4), 521-545. 
Departamento Administrativo Nacional de Estadística [DANE]. (2018). Pobreza monetaria por departamentos. https://www.dane.gov.co/files/investigaciones/condiciones_vida/ pobreza/2018/bt_pobreza_monetaria_18_departamentos.pdf

Departamento Administrativo Nacional de Estadística [DANE]. (2020, 30 de septiembre). DANE Información para todos. https://www.dane.gov.co/index.php/estadisticas-por-tema/ mercado-laboral/empleo-y-desempleo

ESLAVA, M. y ISAACS, M. (2020, 7 de abril). Vulnerabilidad del empleo a la emergencia de COVID-19 en Colombia. https://economia.uniandes.edu.co/images/archivos/pdfs/cede/ Observatorio-coyuntura/Gr\%C3\%A1ficos_Empleos_-_Nacional-07042020_-_1.pdf

GARAY, L. y EsPitiA, J. (2020, 13 de mayo). Impoverishment and exacerbation of inequalities make a basic income in Colombia necessary. UN Periódico Digital. http://unperiodico.unal. edu.co/pages/detail/impoverishment-and-exacerbation-of-inequalities-make-a-basicincome-in-colombia-necessary/

García, M. (2005). Minimum income policies to combat poverty: Local practices and social justice in the "European social model". En Cities of Europe: Changing contexts, local arragements and the challenge to urban cobesion (pp. 301-324). Malden: Blackwell publishing.

Gentilini, U., GROSH, M., RigOlini, J. y Yemtsov, R. (2020). Exploring universal basic income: A guide to navigating concepts, evidence, and practices. Washington: World Bank. https://openknowledge.worldbank.org/handle/10986/32677

Hoynes, H. y Rothstein, J. (2019). Universal Basic Income in the United States and Advanced Countries. Annual Review of Economics, 11, 929-958.

Lamprea, T., Ospina, V., Hernández, G. y Rivera, A. (2020, 27 de mayo). Una medida de los efectos potenciales del covid-19 en el empleo: el caso de la política de aislamiento preventivo obligatorio en Colombia. https://colaboracion.dnp.gov.co/CDT/Estudios $\% 20$ Econmicos/508.pdf

Observatorio Fiscal de la Pontificia Universidad Javeriana. (2020, 14 de junio). El costo y la factibilidad de la renta básica en Colombia. https://c899837a-b1ec-41b5-9d46-3e957755d77b. filesusr.com/ugd/e33cdb_56c9535839004412afa74a4713c0e0df.pdf

Organización para la Cooperación y el Desarrollo Económico (OCDE). (2019). Estadísticas tributarias en América Latina y el Caribe. OCDE. https://www.oecd.org/tax/tax-policy/ brochure-estadisticas-tributarias-en-america-latina-y-el-caribe-2019.pdf

PHELPS, E. (1994). Low-wage employment subsidies versus the welfare state. The American Economic Review, 84(2), 54-58.

Presidencia de la República de Colombia. (2020, 17 de marzo). DAPRE. https://dapre.presidencia.gov.co/normativa/normativa/DECRETO\%20417\%20DEL\%2017\%20DE\%20MARZO\%20 DE\%202020.pdf

Presidencia de la República. (2020, 22 de marzo). DAPRE. https://dapre.presidencia.gov.co/normativa/normativa/DECRETO\%20457\%20DEL\%2022\%20DE\%20MARZO\%20DE\%202020.pdf

RAMíREZ Bonilla, M. C. (2020). El FMI recortó proyección de crecimiento para Colombia de $3,6 \%$ a 3,5\% en 2020. Diario La República. https://www.larepublica.co/globoeconomia/ el-fmi-recorto-su-proyeccion-de-crecimiento-para-colombia-de-36-a-35-para-esteano-2958042 
Revista Dinero. (2020). ¿Qué tan viable sería una renta básica en Colombia? https://www.dinero.com/economia/articulo/que-tan-viable-seria-una-renta-basica-en-colombia/289678

SÁNCHEZ, R. y ChAPARRO, S. (2020). Un piso de protección social para preservar la vida: informalidad, pobreza y vulnerabilidad en tiempos de covid-19. Bogotá: Universidad Nacional de Colombia.

Tena Camporesi, A. (2018). La renta básica universal basada en la evidencia. Política y Sociedad, 851-871. https://doi.org/10.5209/POSO.58497

Universidad de los Andes. (2020a, 1. ${ }^{\circ}$ de octubre). La afectación efectiva del empleo en la pandemia: ¿qué más nos pasó? Nota Macroeconómica n. ${ }^{\circ}$ 26. https://economia.uniandes. edu.co/components/com_booklibrary/ebooks/BM\%2026.pdf

Universidad de los Andes. (2020b, 24 de marzo). Transferencia monetaria inmediata para los informales. Nota Macroeconómica n. ${ }^{\circ}$ 9. https://repositorio.uniandes.edu.co/bitstream/ handle/1992/41149/nota- macroeconomica- 09 . pdf? sequence $=1$ \& is Allowed $=y$

Universidad de los Andes. (2020c, 13 de julio). Ingreso básico garantizado (IBG): ¿es el momento ideal de implementarlo? https://economia.uniandes.edu.co/components/com_booklibrary/ ebooks/BM\%2022.pdf

Universidad Johns Hopkins. (2020, 16 de octubre). ESRI Environmental Systems Research Institute. https://www.arcgis.com/apps/MapSeries/index.html?appid=c8fee09df07f $49 \mathrm{f}$ 1aaf4fof086bf7d8a

VAN PARIJS, P. (1992). Competing justifications of basic income. En P. VAN PARIJS, Arguing for basic income: Ethical foundations for a radical reform (pp. 3-46). Londres: Verso.

Van Parijs, P. y Vanderborght, Y. (2015). La renta básica. Ariel. 